\title{
Extinction after water and intracranial reinforcement*
}

\author{
GERALD L. WEST \\ University of Oklahoma Medical Center, Oklahoma City, Okla. 73104 \\ and \\ LARRY D. REID \\ Bradley University, Peoria, Ill. 61606
}

Three groups of four albino rats were compared during extinction of a tube-contact response for intracranial reinforcement (ICR) and for water reinforcement (WR). One ICR group (ICR-D) showed a 12 -h performance decrement and the other group (ICR-N) showed no decrement in a runway situation. In general, there were no differences between the three groups during extinction after various delays between a 2 -min preextinction base and extinction, except that on one of three extinction criteria, WR Ss extinguished more rapidly than the ICR Ss. The results provided evidence to contradict Deutsch's drive decay theory of ICR and provided support for theories that assume that ICR mimics conventional reinforcement. It was concluded that Deutsch's theory is an overgeneralization and does not apply to all Ss working for brain stimulation.

Deutsch (Deutsch \& Deutsch, 1966) has offered a drive decay theory to explain certain "anomalous" behaviors that sometimes accompany brain stimulation reward, intracranial reinforcement (ICR). Briefly, those anomalies are (1) Ss need to be "primed" before they will begin working for ICR, (2) Ss do not satiate when working for ICR, unlike Ss working for conventional reinforcement, e.g., food or water, and (3) extinction after ICR is much more rapid than after conventional reinforcement and is a function of time since last reinforcement, whereas extinction after conventional reinforcement is a function of the number of unreinforced responses. There are several studies from which Deutsch has drawn support for his theory (Deutsch \& Howarth, 1963; Gallistel, 1964, 1969; Trowill, Panksepp, \& Gandleman, 1969), but the one that is most important here is a study which provided specific support for the theory via extinction performance after ICR (Howarth \& Deutsch, 1962). The conclusions of the Howarth and Deutsch study were that ICR Ss perform differently from conventional Ss and that, in keeping with the third anomaly mentioned above, extinction after ICR is a function of time. At this point, it is interesting to note two facts. First, conventionally rewarded Ss were not

*Tlis research is part of a thesis presented to the Department of Psychology, Bradley University, in partial fulfillment of requirements for the $M A$ degree by $G$. $L$. West, supervised by L. D. Reid. Support was provided by Bradley University, Faculty Research Committee, and by The University of Oklahoma Medical Center, Biological Psychology, Behavioral Sciences Research Training Grant MH-10322-07. compared directly with ICR Ss. Rather, Deutsch drew upon conclusions about extinction performance of conventionally rewarded Ss that had been run in Skinner boxes. Second, and most important, ICR Ss received reinforcement during an operant (i.e., during a leverpress) while conventionally rewarded Ss received reinforcement after an operant (i.e., during a consummatory response that followed a leverpress). Gibson, Sakai, Reid, \& Porter (1965) demonstrated that when the two types of reinforcement are placed in the same temporal relationship with the operant, ICR Ss perform indistinguishably from conventionally reinforced Ss. They compared the two types of reinforcement directly. The Gibson et al study was criticized by Deutsch \& DiCara (1967), however, because both the ICR and the conventional Ss had been food deprived. The criticism was that the food deprivation, rather than the ICR itself, may have been the determiner of the ICR Ss' performance during extinction.

A further complication regarding Deutsch's theory is that ICR Ss do not all perform the same for ICR. One such measure is obtained in a runway situation (i.e., a T-maze, straight-alley maze, or C-maze). In such a situation, some ICR Ss perform similarly to conventional Ss. That is, they run fast on the first trial after a period of no ICR. Other ICR Ss perform anomalously. That is, they run slowly, or not at all, on the first trial, but may run quite fast on subsequent trials after receiving reinforcement. The former type of $\mathrm{S}$ has been referred to as nondecrement (ICR-N) and the latter as overnight (or over intersession interval) decrement (ICR-D) (Wasden, Reid, \& Porter, 1965). It is possible that some of the differences between conventional and ICR Ss predicted by Deutsch's theory are due to the use of only, say, decrement Ss.

In the following study, each of the above-mentioned factors was taken into account in a reexamination of the Howarth \& Deutsch (1962) study. Two groups of ICR Ss, decrement and nondecrement, were compared to a water reward (WR) control group. Reinforcement for all Ss was presented during contact with a drinking tube (a consummatory-o perant response). Finally, in light of Deutsch \& DiCara's (1967) criticisms of the Gibson et al study, WR Ss were water deprived and the ICR Ss were not deprived. A 2-min preextinction base and three extinction criteria were used. Howarth \& Deutsch (1962) used as their extinction criterion a 90-deg turn from the manipulandum, while Gibson et al used the total number of responses in a $30-\mathrm{min}$ period of extinction. A priori, the 90-deg turn seems inappropriate, since Ss working for conventional reinforcement and for ICR often make such turns, even when reinforcement is available with each response. Therefore, in addition to the 90-deg turn and the total number of responses in $15 \mathrm{~min}$ (rather than $30 \mathrm{~min}$ ), a third criterion, a $1.5-\mathrm{min}$ period of no responding, was used. Both time and number of responses were recorded to the 90-deg turn and to $1.5 \mathrm{~min}$ of no responding.

\section{METHOD}

Twelve adult male albino rats were divided into three groups of four. The WR Ss were unoperated and served as conventional reward controls. The ICR Ss were each fixed with a chronic bipolar lateral hypothalamic electrode (MS-303-018-312-SS-008, Plastic Products Co., Roanoke, Va.), insulated except at the cross sections of their tips. The ICR-N group were those Ss that ran the first trial in a C-maze in less than $15 \mathrm{sec}$ after $12 \mathrm{~h}$ of no ICR. The ICR-D group were those Ss that ran in more than $15 \mathrm{sec}$. A trial consisted of running the length of the maze for the opportunity to press a lever for ICR $(60-\mathrm{Hz}$ sine waves of up to $.5 \mathrm{sec}$ duration with each leverpress, intensities ranging from 15 to 60 micro $\mathrm{A}$ across Ss). For details of the C-maze procedure, see West, Hunsicker, \& Reid (1971). All Ss pressed rapidly without priming when placed in front of the lever in the goalbox. The WR Ss were not run in the C-maze.

Extinction testing was performed in a small Plexiglas chamber, $24 \times 21 \times 20 \mathrm{~cm}$. The schedule of testing was identical for all groups 


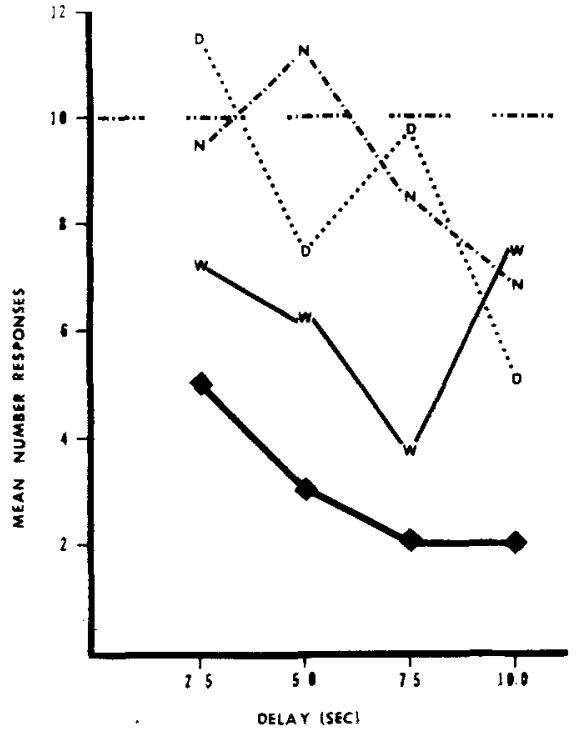

Fig. 1. Mean responses to a 90-deg turn from the tube, with each delay, averaged across 4 days of extinction. Horizontal line and heavy curve are from Howarth \& Deutsch (1962).

except that the WR Ss were $24 \mathrm{~h}$ water deprived. ICR was the same as in the C-maze except that a drinking tube was the manipulandum rather than a lever. WR Ss could obtain water with each lick of the tube, which was inserted through a small hole in one of the chamber walls. During two daily 5 -min training sessions and during the 2 -min preextinction base sessions of testing, reinforcement was available with each contact with the tube. During extinction, a "dry" tube was inserted, from which no reinforcement $w$ as a vailable. Ss were assigned randomly, one from each group, to one of four sequences of delay presentations, arranged in the form of a cyclic Latin square (2.5 to $10.0 \mathrm{sec}$; 5.0 to $2.5 \mathrm{sec} ; 7.5$ to $5.0 \mathrm{sec}$; and 10.0 to $7.5 \mathrm{sec}$ ). Thus, one $S$ from each group received each delay on each of 4 days of testing. An $S$ received one delay per session, one session per day, until each $\mathrm{S}$ had been tested with each delay. At the end of the 2-min preextinction base, the tube was withdrawn for a delay of $2.5,5.0,7.5$, or $10.0 \mathrm{sec}$ and then replaced by the "dry" tube. The measures taken during extinction were (1) time and (2) number of contacts to a 90-deg turn from the tube, (3) time and (4) number of contacts to a $1.5-\mathrm{min}$ period of no contacts, and (5) total number of contacts in $15 \mathrm{~min}$ of extinction.

\section{RESULTS AND DISCUSSION}

All electrode tips were determined histologically to lie deep within the lateral hypothalamic area and most likely stimulated fibers of the medial forebrain bundle, zona incerta, Forel's fields, crus cerebri, internal capsule, and optic tract. There were no obvious relationships between $\mathrm{C}$-maze performance (i.e., whether decrement or nondecrement) and sites of stimulation.

A rank order correlation revealed highly significant correlations between time and number of contacts to a 90-deg turn (rho $=.90$ ) and to $1.5 \mathrm{~min}$ of no responding (rho $=.91$ ). Therefore, only the number of contacts to each criterion were reported.

The four measures, contacts during 2 min of preextinction base, contacts to a 90-deg turn, contacts to a $1.5-\mathrm{min}$ period of no responding, and total number of contacts in $15 \mathrm{~min}$ of extinction, were analyzed with a random blocks factorial analysis of variance (Kirk, 1969 , p. 239). This made possible an analysis of the magnitude of differences between groups and between sequences (order of delays) without confounding due to days (multiple testing). Student ts were performed where indicated.

The groups differed markedly in responding: during the 2 -min preextinction base, $F(2,33)=32.64$, $\mathrm{p}<.01$. The WR Ss responded more than the combined ICR groups, $t(45)=6.18, p<.01$. The ICR-D Ss responded more than did the ICR-N Ss, $\mathrm{t}(30)=4.42, \mathrm{p}<.01$.

Figure 1 depicts the group mean contacts to a 90-deg turn after each delay. The horizontal broken line and the heavy curve represent, respectively, the hypothetical base and number of contacts to a 90-deg turn after each delay, as predicted from Deutsch's theory (Howarth \& Deutsch, 1962). The WR, ICR-N, and ICR-D groups are represented by $\mathrm{W}, \mathrm{N}$, and $D$, respectively. The only difference that was reliable for this measure was across days, $F(3,33)=3.91, p<.05$. All Ss responded less on the fourth day than on the first, $t(21)=3.17$, $\mathrm{p}<.01$, indicating that Ss learned to respond less during extinction over multiple testing. The fact that there were no reliable differences between groups, however, indicated that either the criterion was not sensitive to differences in performance, or that, in fact, ICR Ss do not perform differently from conventional Ss under the conditions of the experiment.

From Fig. 1, it appears that the groups tended to follow Deutsch's theoretical curve. Such was not the case, however, as indicated by an unreliable $\mathrm{F}$ for differences between sequences on the 90-deg turn criterion. In addition, when the data were collapsed over all other variables, there were no reliable differences in performance between the four delays (all ts, $p>.05$ ). The latter applied to each of the criteria. The 90-deg turn criterion must be an inappropriate one, since, as predicted a priori, Ss were frequently observed to make such turns during the 2-min preextinction base when reinforcement was available with each contact.

On the 1.5 -min criterion, the groups differed reliably, $F(2,33)=5.14$, $\mathrm{p}<.01$, and all $\mathrm{Ss}$ responded differently across days, $F(3,33)=5.36, \quad p<.01$. The WR group responded less than the combined ICR Ss, $t(45)=2.57$ $\mathrm{p}<.01$, but there was no reliable difference between the two ICR groups. All groups responded less on the fourth day than on the first, $t(21)=3.58, p<.01$. Once again, no support was found for Deutsch's drive decay theory. The WR Ss responded less than the ICR Ss, just the opposite of what the theory would have predicted. A possible explanation for such a result is that ICR is simply a more potent reinforcer than water, even after 24 h of deprivation.

Performance during extinction was indistinguishable between groups and sequences, and across days on the basis of the number of contacts during $15 \mathrm{~min}$ of extinction. This result complies with the Gibson et al (1965) results.

Figure 2 depicts the data from only the first day of extinction and of preextinction base, with $\mathrm{n}=1$ for each point on the graphs. Notice that, in general, the data of the first day reflect quite well the data for all 4 days. Only on the $1.5-\mathrm{min}$ criterion did the groups differ reliably, with the WR Ss responding less than the combined ICR Ss, $t(9)=3.67, \mathrm{p}<.01$. The difference is amplified considerably by the fact that the WR Ss responded more during the 2-min base than did the combined ICR Ss, $t(9)=4.01, \quad p<.01$. According to Deutsch's theory, the WR Ss should have responded more during extinction on this criterion than the ICR Ss, but, in fact, the exact opposite was the case. In terms of Deutsch's theory, the conventional controls showed more "drive decay" than the ICR Ss.

\section{CONCLUSIONS}

The most obvious conclusion that may be drawn from these results is that under the appropriate conditions extinction after ICR is indistinguishable from that after conventional reinforcement (WR). The implication is that brain stimulation reward is not peculiar when compared to conventional reward, and may therefore be used to study, quite directly, the nature of 


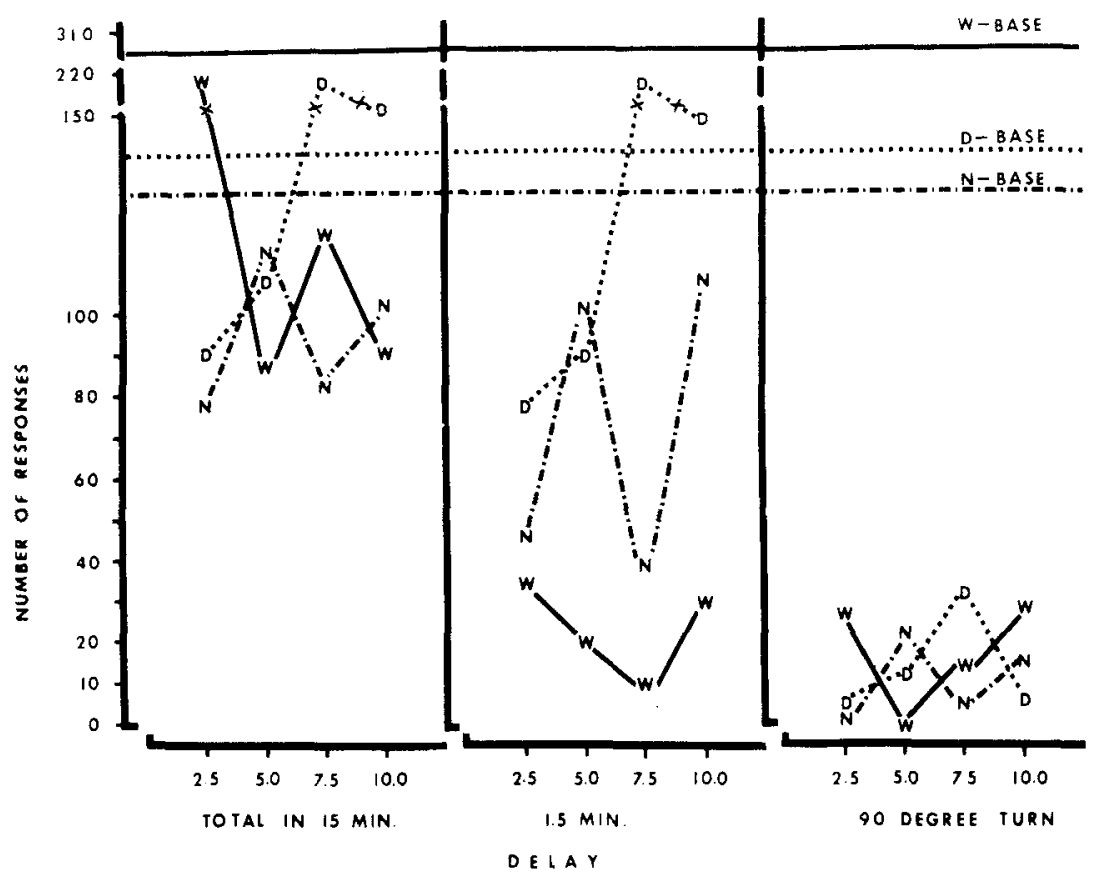

Fig. 2. Number of responses to each criterion for each delay on the first day of extinction $(n=1) . W=$ water $\operatorname{reward}(W R), N=I C R-N$, and $D=I C R-D$.

reinforcement. This provides support for theories that are built on the assumption that ICR mimics conventional reinforcement (Olds, 1969 ; Stein \& Wise, 1971). It also provides evidence against theories that are built on the assumption that ICR is peculiar or "anomalous" (Deutsch \& Deutsch, 1966; Gallistel, 1969).

A possible salvation of Deutsch's drive decay theory of ICR is that it may explain the behavior of certain Ss working for ICR. That is, some Ss will not press a lever for ICR until they have received free trains (priming) of ICR. The Ss of the present study, however, did not require priming. As mentioned earlier, they all pressed a lever for ICR rapidly when placed in front of it, whether or not they ran the maze. It is certainly possible that Deutsch's explanation will hold with Ss that show all the anomalies that he has described. Not all Ss show those anomalies, however, and therein lies theory. Insofar as Deutsch has implied that his theory holds for all Ss working for ICR, he has made a gross the difficulty with the drive decay overgeneralization.

\section{REFERENCES}

DEUTSCH, J. A., \& DEUTSCH, D. Physiological psychology. Homewood, Ill: Dorsey, 1966.

DEUTSCH, J. A., \& DiCARA, L. Hunger and extinction in intracranial self-stimulation. Journal of Comparative \& Physiological Psychology, 1967,63 $34 \div-347$.

DEUTSCH, J. A., \& HOWARTH, C. I. Some tests of a theory of intracranial self-stimulation. Psychological Review, $1963,70,446-460$.

GALIISTEL, C R. Electrical self-stimulation and its theoretical implications. Psychological Bulletin, $1964,61,23-24$.

GALLISTEL, $C$. $R$. The incentive of brain-stimulation reward, Journal of Comparative \& Physiological Psychology, $1969,69,713-721$.

GIBSON, W. E.. SAKAI, M., REID, L. D., \& P ORTER. P. B. Intracranial reinforcement compared with sugar-water reinforcement. Science, 1965,148 , 1357-1359.

HOWARTH, C, I \& DEUTSCH, J A. Drive decay: The cause of fast "extinction" of habits learned for brain-stimulation. Science, 1962, 137, 35-36.

KIRK, R. E. Experimental design: Procedures for the behavioral sciences. Belmont, Calif: Brooks/Cole, 1969.

OLDS, J. The central nervous system and reinforcement of behavior. American Psy chologist, 1969, 24, 114-132.

STEIN, L.. \& WISE, C. D. Possible etiology of schizophrenia: Progressive damage to the noradrenergic reward system by 6-hydrox y dopamine. Science, 1971, 171 , 1032-1036.

TROWILL, J. A., PANKSEPP, J., \& GANDLEMAN, R. An incentive model of rewarding brain stimulation. Psychological Review, 1969, 76, 264-281.

WASDEN, R. E., REID, L. D., \& PORTER, $P$. B. Overnight performance decrement with intracranial reinforcement. Psychological Reports, 1965, 16, 653-658.

WEST, G. L., HUNSICKER, J. P., \& REID, L. D. Performance differences for intracranial reinforcement as a function of recency and number of stimulations. Communications in Behavioral Biology 1971, 7, 171-176, Abstract No. 05710024 . 